\title{
Kennzahlen für die Zielkostenkontrolle
}

\section{Rolf Brühl}

Online publiziert: 19. Februar 2010

(C) Springer-Verlag 2010

Zusammenfassung In diesem Artikel wird die Entwicklung verschiedener Kennzahlen für die Zielkostenkontrolle und mit ihr verbundener Visualisierungsdiagramme nachgezeichnet. Die schon früh geäußerten Bedenken gegen den Zielkostenindex werden systematisiert; es wird dabei gezeigt, dass er in bestimmten Intervallen systematisch zu Fehlinterpretationen verleitet. Daher wird dafür plädiert, ihn in der Zielkostenkontrolle nicht mehr zu verwenden. Besondere Beachtung findet eine Variante auf Basis von Kostenbeträgen, die als „relative Zielkostenabweichung“ bezeichnet wird. Es wird gezeigt, dass diese Kennzahl den Rechnungszweck, Kontrollinformationen zu generieren, erfüllt und wie ein entsprechendes Zielkostenkontrolldiagramm konstruiert wird. Abschließend werden die wesentlichen Kennzahlen für die Zielkostenkontrolle vergleichend diskutiert.

Schlüsselwörter Relative Zielkostenabweichung · Zielkostenindex ·

Zielkostenkontrolldiagramm

\section{Einleitung}

Die Zielkostenplanung und -kontrolle gehört in vielen Unternehmen zu den zentralen Aufgaben des Kostenmanagements, mit deren Hilfe Kosteninformationen schon möglichst früh im Produktlebenszyklus insbesondere für die Produktentwicklung und -konstruktion zur Verfügung gestellt werden sollen (vgl. Franz u. Kajüter 2002, S. 579 ff.; Kajüter 2005, S. 91 ff.; zu aktuellen Forschungsüberblicken vgl. Ansari et al. 2007; Götze u. Linke 2008). Zu den zentralen Elementen ihres Vorgehens gehören:

Prof. Dr. R. Brühl (凶)

Lehrstuhl für Unternehmensplanung und Controlling, ESCP Europe Wirtschaftshochschule Berlin, Heubnerweg 6, 14059 Berlin, Deutschland

e-mail: rolf.bruehl@escpeurope.de 
1. die Bestimmung des Zielpreises und des Zielgewinns zur Festlegung der Zielkosten, 2. die Bestimmung der Bedeutung der Komponenten sowie 3. die Berechnung des Zielkostenindex und seine graphische Darstellung in einem Zielkostenkontrolldiagramm. Letztere Informationen sollen Handlungsbedarf anzeigen und Hinweise geben, bei welchen Komponenten Kosten reduziert oder die Qualität verbessert werden soll.

In diesem Artikel wird sich auf das dritte Element konzentriert, indem absolute und relative Kennzahlen für die Zielkostenkontrolle sowie die entsprechenden Zielkostenkontrolldiagramme analysiert werden. Er gliedert sich wie folgt: In einem ersten Schritt werden die in der Zielkostenkontrolle verfolgten zwei Rechnungszwecke - Kontrolle der Zielkostenerreichung und Kontrolle des Kosten-NutzenVerhältnisses - dargestellt. Im dritten Kapitel werden die Schwächen des Zielkostenindex aufgezeigt und anschließend werden die vorgeschlagenen Modifikationen des Zielkostenindex diskutiert. Im vierten Kapitel wird die Kennzahl „relative Zielkostenabweichung" vorgestellt und ein Zielkostenkontrolldiagramm auf ihrer Basis entwickelt. Im abschließenden fünften Kapitel wird über die verschiedenen Kennzahlen für die Zielkostenkontrolle diskutiert und ein konsistentes Kennzahlensystem für die Zielkostenkontrolle vorgestellt.

\section{Rechnungszwecke der Zielkostenkontrolle}

Kontrollrechnungen liefern Informationen, um analysieren zu können, ob Ziele erreicht oder ob sie verfehlt werden. In der Zielkostenkontrolle sind die Zielkosten, die als Kostenobergrenze zu interpretieren sind, zu erreichen: Jede Abweichung von diesem Ziel führt unter der Annahme von nicht zu verändernden Zielpreisen zu Gewinneinbußen. Als wichtige Information wird daher die Zielkostenabweichung des Produkts $\left(\Delta k_{Z, P} ; G E / P E\right)^{1}$ ermittelt (vgl. Cooper u. Slagmulder 2005, S. 254):

$$
\Delta k_{Z, P}=k_{P, P}-k_{Z, P}
$$

Sie vermittelt unter der Annahme, dass die Plankosten des Produkts ${ }^{2}\left(k_{P, P} ; G E / P E\right)$ über den Zielkosten des Produkts $\left(k_{Z, P}, G E / P E\right)$ liegen, den Kostenreduktionsbedarf. Da es im Zielkostenmanagement um die Steuerung der Produktentwicklung geht, wird in einem zweiten Schritt die Zielkostenabweichung je Komponente $\left(\Delta k_{Z, K} ; G E / K E\right)$ berechnet. $^{3}$

$$
\Delta k_{Z, K}=k_{P, K}-k_{Z, K}
$$

\footnotetext{
${ }^{1}$ Zur Bezeichnung von Dimensionen werden folgende Abkürzungen verwendet: $G=$ Geld, $E=$ Einheit, $P=$ Produkt, $K=$ Komponente. Wird keine Dimension angegeben, handelt es sich um eine dimensionslose Größe.

${ }^{2}$ Alternative Bezeichnungen für Plankosten sind Schätz-, Standardkosten oder Drifting Costs.

${ }^{3}$ Im Folgenden wird zur Vereinfachung nur die Komponentenmethode betrachtet: Alle Aussagen dieses Aufsatzes lassen sich allerdings problemlos auf die Funktionsmethode übertragen (vgl. zu dieser Unterscheidung Tanaka 1989, S. 52 f.).
} 
Die Plankosten einer Komponente $\left(k_{P, K} ; G E / K E\right)$ werden aus Schätzungen auf Basis der vorhandenen Technologie ermittelt. Die Zielkosten je Komponente $\left(k_{Z, K}\right.$; $G E / K E$ ) ergeben sich aus der Multiplikation der Bedeutung einer einzelnen Komponente ${ }^{4}$ für das Produkt $\left(b_{k}\right)$ mit den Zielkosten des Produkts.

$$
k_{Z, K}=b_{K} \cdot k_{Z, P}
$$

Damit ist ein erster wesentlicher Rechnungszweck konstatiert, der sich auf zwei Kontrollobjekte bezieht: Es sollen die Abweichungen von den Zielkosten des Produkts insgesamt und für jede einzelne Komponente separat ermittelt werden. Es ist jedoch ein zweiter Rechnungszweck zu beachten: die Betrachtung der Kosten-NutzenRelation der einzelnen Komponenten (vgl. Fischer u. Schmitz 1994, S. 427; Mussnig 2001, S. 268). Sie wird durch den Zielkostenindex $\left(Z I_{K}\right)$ ausgedrückt, der als Quotient die Plankostenanteile der Komponente $\left(a_{K}\right)$ und die Bedeutung der Komponente $\left(b_{K}\right)$, die ihrem Anteil an den Zielkosten des Produkts entspricht, ins Verhältnis setzt (vgl. Tanaka 1989, S. 61; Schweitzer u. Küpper 2008, S. 713).

$$
Z I_{K}=\frac{a_{K}}{b_{K}} \quad \text { wobei } a_{K}=\frac{k_{P, K}}{k_{P, P}}
$$

Der Zielkostenindex wird in zwei Varianten interpretiert. Wie Formel (3) zeigt, ist die Bedeutung $b_{K}$ gleich dem relativen Anteil der Zielkosten der Komponente an den Zielkosten des Produkts $\left(k_{Z, K} / k_{Z, P}\right)$ : Der Zielkostenindex setzt daher erstens zwei Kostenanteile ins Verhältnis. In der zweiten Interpretation wird der Kosten-NutzenAspekt von $b_{K}$ betont und vom Verhältnis der Plankosten zur Bedeutung der Komponente gesprochen.

\section{Der Zielkostenindex und seine Modifikationen}

Wenn mit dem Zielkostenindex das Kosten-Nutzen-Verhältnis des zu entwickelnden Produktes beurteilt werden soll, sind drei Fälle zu unterscheiden (vgl. Freidank u. Zaeh 1997, S. 252): 1. Im idealen Fall ist der Zielkostenindex gleich 1. Er gilt als idealtypischer Wert, für den angenommen wird, dass sich der Nutzen für den Kunden und die dafür durch das Unternehmen eingesetzten Ressourcen genau entsprechen. Tritt dieser Fall für eine Komponente auf, besteht aus Sicht des Unternehmens kein Handlungsbedarf für diese Komponente. 2. Bei einem Zielkostenindex größer als 1 ist der Kostenanteil der Komponente größer als ihr Nutzenanteil oder ihre Bedeutung; folglich besteht ein Kostenreduktionsbedarf. 3. Ist der Zielkostenindex hingegen kleiner als 1, wird angenommen, dass der Wert einer Komponente zu gering ist, z. B. aufgrund qualitativ minderwertigen Materials. Eventuell können noch weitere Ressourcen für sie eingesetzt werden, d. h., es besteht mithin Qualitätsverbesserungsbedarf.

\footnotetext{
${ }^{4}$ Die Bedeutung der Komponente soll den Kundennutzen ausdrücken. Sie wird mithilfe der Komponentenmethode durch die Multiplikation von zwei Größen berechnet: 1. die Gewichtung der Funktionen, die der Kunde mit dem Produkt verbindet, durch die Kunden und 2. den Anteil, den eine Komponente des Produkts an der Funktionserfüllung hat (vgl. z. B. Götze 2007, S. 280).
} 
Tab. 1 Zahlenbeispiel zum Zielkostenindex

\begin{tabular}{|c|c|c|c|c|c|c|}
\hline & $\begin{array}{l}\text { Kosten- } \\
\text { anteil }\left(a_{K}\right)\end{array}$ & $\begin{array}{l}\text { Bedeutung } \\
\left(b_{K}\right)\end{array}$ & $\begin{array}{l}\text { Zielkosten- } \\
\text { index }\left(Z I_{K}\right)\end{array}$ & $\begin{array}{l}\text { Plankosten } \\
\left(k_{P, K} ;\right. \\
G E / K E)\end{array}$ & $\begin{array}{l}\text { Zielkosten } \\
\left(k_{Z, K} ;\right. \\
G E / K E)\end{array}$ & $\begin{array}{l}\text { Zielkosten- } \\
\text { abweichung } \\
\left(\Delta k_{Z, K} ; G E / K E\right)\end{array}$ \\
\hline A & 0,23 & 0,25 & $\begin{array}{l}0,92 \\
(=0,23 / 0,25)\end{array}$ & $\begin{array}{l}138,00 \\
(=0,23 \cdot 600)\end{array}$ & $\begin{array}{l}112,50 \\
(=0,25 \cdot 450)\end{array}$ & $\begin{array}{l}25,50 \\
(=138-112,50)\end{array}$ \\
\hline B & 0,47 & 0,50 & $\begin{array}{l}0,94 \\
(=0,47 / 0,50)\end{array}$ & $\begin{array}{l}282,00 \\
(=0,47 \cdot 600)\end{array}$ & $\begin{array}{l}225,00 \\
(=0,50 \cdot 450)\end{array}$ & $\begin{array}{l}57,00 \\
(=282-225)\end{array}$ \\
\hline $\mathrm{C}$ & 0,30 & 0,25 & $\begin{array}{l}1,20 \\
(=0,30 / 0,25)\end{array}$ & $\begin{array}{l}180,00 \\
(=0,30 \cdot 600)\end{array}$ & $\begin{array}{l}112,50 \\
(=0,25 \cdot 450)\end{array}$ & $\begin{array}{l}67,50 \\
(=180-112,50)\end{array}$ \\
\hline Summe & 1,00 & 1,00 & & $\begin{array}{l}600,00 \\
\left(k_{P, P} ; G E / P E\right)\end{array}$ & $\begin{array}{l}450,00 \\
\left(k_{Z, P} ; G E / P E\right)\end{array}$ & $\begin{array}{l}150,00 \\
\left(\Delta k_{Z, P} ; G E / P E\right)\end{array}$ \\
\hline
\end{tabular}

Liegen die Zielkosten unter den Plankosten, erzeugt der Zielkostenindex allerdings Informationen, die zu Fehlinterpretationen verleiten. Dies lässt sich am einfachsten an einem fiktiven Zahlenbeispiel zeigen. Gegenstand der Zielkostenkontrolle ist ein Produkt mit drei Komponenten A, B und C, für das Zielkosten von 450 $(G E / P E)$ und Plankosten von $600(G E / P E)$ ermittelt werden (weitere Angaben sind Tab. 1 zu entnehmen).

Aus der Tabelle ist das Interpretationsprobleme des Zielkostenindex unmittelbar zu erkennen. Der Zielkostenindex weist bei den Komponenten A und B in die falsche Richtung, denn er zeigt einen Qualitätsverbesserungsbedarf an, obwohl die Zielkostenabweichung (in der letzten Spalte von Tab. 1) ausweist, dass tatsächlich für beide Komponenten ein Kostenreduktionsbedarf besteht. Bevor dies näher analysiert wird, soll das Beispiel noch visualisiert werden.

Die Visualisierung der Komponenten wird in einem Zielkostenkontrolldiagramm vorgenommen, indem auf der $x$-Achse die Bedeutung $\left(b_{K}\right)$ und auf der $y$-Achse der Plankostenanteil $\left(a_{K}\right)$ abgetragen wird: So lässt sich jeder Komponente im Koordinatensystem ein Punkt zuordnen. Zusätzlich werden weitere Elemente in das Diagramm eingefügt: erstens die Ideallinie, in der sich Plankostenanteil und Bedeutung entsprechen, und zweitens eine Zielkostenzone mit einer Untergrenze $\left(y_{U G}\right)$ und Obergrenze $\left(y_{O G}\right)$. Die Zielkostenzone stellt einen Toleranzbereich dar, innerhalb dessen Abweichungen von der Ideallinie zugelassen sind. Mit ihrer Hilfe wird das Entscheidungskriterium für die erste Handlungsmöglichkeit modifiziert, weil innerhalb der Grenzen kein Handlungsbedarf angezeigt wird. Sie wird in der Regel so konstruiert, dass sich die Toleranzgrenzen mit steigender Bedeutung immer mehr der Ideallinie nähern (vgl. Tanaka 1985, 1989; Glaser 2006, S. 217).

$$
y_{U G}=\sqrt{\max \left\{b_{K}^{2}-\alpha^{2}, 0\right\}} \text { und } y_{O G}=\sqrt{b_{K}^{2}+\alpha^{2}}
$$

Der Parameter $\alpha$ legt die Toleranz fest, mit der Abweichungen von der Ideallinie akzeptiert werden. Er liegt üblicherweise zwischen 0,1 und 0,2, im Beispiel bei 0,15.

Wenn eine solche Zielkostenzone eingesetzt wird, ist zu bedenken, dass unter der Annahme der Konstanz der Zielpreise jedes Abweichen von den Zielkosten des Produkts den angestrebten Zielgewinn und die damit verbundene Rendite schmälert. Es sollte daher als wichtige Bedingung beachtet werden, dass die Summe der Plankosten 


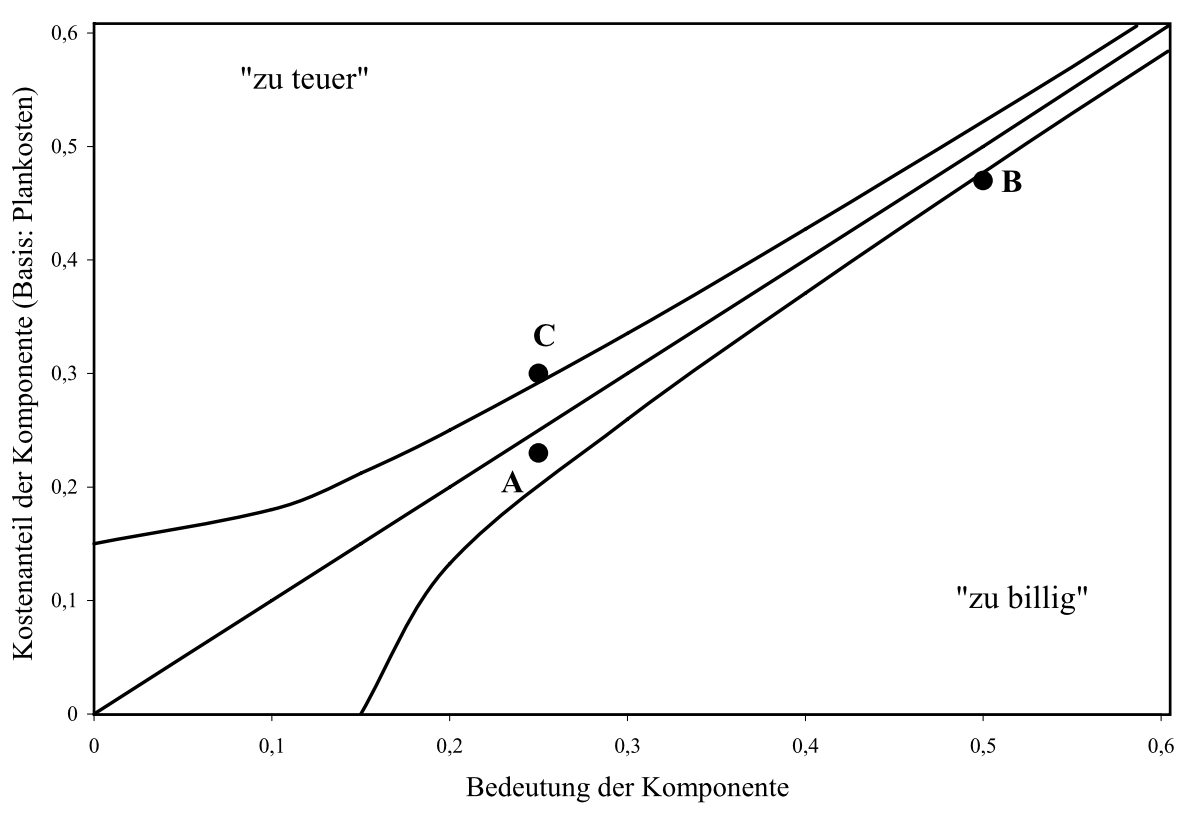

Abb. 1 Zielkostenkontrolldiagramm zum Zielkostenindex

aller Komponenten die Zielkosten des Produkts nicht überschreitet (vgl. Glaser 2002, S. 238; Noltemeier 2003, S. 44).

$$
\sum_{K} k_{P, K} \leq k_{Z, P}
$$

Das Beispiel dient der Illustration des Sachverhalts und kann keine Allgemeingültigkeit beanspruchen. Es lässt sich jedoch leicht zeigen, dass der Zielkostenindex systematisch verzerrte Informationen liefert. Dem Beispiel lässt sich entnehmen, dass das Verhältnis zwischen Ziel- und Plankosten des Produkts 0,75 $(=450 / 600)$ ist. Dieses Verhältnis kann nun verwendet werden, um zu zeigen, in welchen Intervallen der Zielkostenindex systematisch zu Fehlinterpretationen führt. Im Beispiel entsteht das Problem im Intervall zwischen 0,75 und 1: Jeder Zielkostenindex einer Komponente, der größer als 0,75 und kleiner als 1 ist, zeigt in die falsche Richtung. Dass dies generell gilt, lässt sich durch folgende Ungleichung zeigen (vgl. Brühl 2010, S. 10):

$$
\begin{aligned}
& \frac{a_{K}}{b_{K}}>\frac{k_{Z, P}}{k_{P, P}} \quad \text { lässt sich umformen zu } \\
& a_{K} \cdot k_{P, P}>b_{K} \cdot k_{Z, P} \quad \text { oder } \quad k_{P, K}>k_{Z, K}
\end{aligned}
$$

Liegt der Zielkostenindex über dem Verhältnis aus Ziel- und Plankosten des Produkts, gibt es einen Kostenreduktionsbedarf für diese Komponente, da seine Plankosten über den Zielkosten liegen. Da es eine realistische Annahme ist, dass die Zielkosten des Produkts unter den Plankosten des Produkts liegen, wird ihr Quotient in der Regel unter 1 liegen und der Zielkostenindex im Intervall zwischen diesem Quotienten und 
Tab. 2 Interpretation des Zielkostenindex

\begin{tabular}{|c|c|c|}
\hline & Fall $1\left(k_{Z, P}<k_{P, P}\right)$ & Fall $2\left(k_{Z, P}>k_{P, P}\right)$ \\
\hline $\begin{array}{l}\text { Fall a } \\
\frac{a_{K}}{b_{K}}>\frac{k_{Z, P}}{k_{P, P}}\end{array}$ & $\begin{array}{l}\text { Alle Zielkostenindices zwischen } 1 \\
\text { und dem Quotienten aus gesamten } \\
\text { Ziel- und Plankosten zeigen } \\
\text { Qualitätsverbesserungsbedarf an, } \\
\text { obwohl Kostenreduktionsbedarf } \\
\text { besteht. }\end{array}$ & $\begin{array}{l}\text { Der Zielkostenindex zeigt den } \\
\text { korrekten Handlungsbedarf an. }\end{array}$ \\
\hline $\begin{array}{l}\text { Fall b } \\
\frac{a_{K}}{b_{K}}=\frac{k_{Z, P}}{k_{P, P}}\end{array}$ & $\begin{array}{l}\text { Der Zielkostenindex zeigt } \\
\text { Qualitätsverbesserungsbedarf an, } \\
\text { obwohl kein Handlungsbedarf } \\
\text { besteht. }\end{array}$ & $\begin{array}{l}\text { Der Zielkostenindex zeigt } \\
\text { Kostenreduktionsbedarf an, obwohl } \\
\text { kein Handlungsbedarf besteht. }\end{array}$ \\
\hline $\begin{array}{l}\text { Fall c } \\
\frac{a_{K}}{b_{K}}<\frac{k_{Z, P}}{k_{P, P}}\end{array}$ & $\begin{array}{l}\text { Der Zielkostenindex zeigt den } \\
\text { korrekten Handlungsbedarf an. }\end{array}$ & $\begin{array}{l}\text { Alle Zielkostenindices zwischen } 1 \text { und } \\
\text { dem Quotienten aus gesamten Ziel- } \\
\text { und Plankosten zeigen } \\
\text { Kostenreduktionsbedarf an, obwohl } \\
\text { Qualitätsverbesserungsbedarf besteht. }\end{array}$ \\
\hline
\end{tabular}

dem Idealwert in die falsche Richtung zeigen (Fall 1a in Tab. 2). Je größer die Differenz zwischen Zielkosten und Plankosten ist, desto kleiner ist dieser Quotient und umso größer sind die Möglichkeiten des Zielkostenindex, zu Fehlinterpretation zu verleiten.

Bisher wird von dem typischen Fall ausgegangen, dass die Zielkosten kleiner als die Plankosten sind. Der Zielkostenindex zeigt aber auch für den Fall, dass die Plankosten kleiner als die Zielkosten sind, in einem Intervall systematisch in die falsche Richtung.

$$
\begin{aligned}
& \frac{a_{K}}{b_{K}}<\frac{k_{Z, P}}{k_{P, P}} \text { lässt sich umformen zu } \\
& a_{K} \cdot k_{P, P}<b_{K} \cdot k_{Z, P} \quad \text { oder } \quad k_{P, K}<k_{Z, K}
\end{aligned}
$$

Wenn der Quotient aus Ziel- und Plankosten des Produkts größer ist als der Zielkostenindex, dann sind die Zielkosten der Komponente größer als die Plankosten der Komponente: Es besteht die Möglichkeit einer Qualitätsverbesserung. Liegt der Zielkostenindex jedoch zwischen 1 und dem Quotienten aus Ziel- und Plankosten des Produkts, veranlasst er Fehlinterpretationen, da er Kostenreduktionsbedarf anzeigt, obwohl eine Qualitätsverbesserung möglich ist (Fall 2c in Tab. 2).

In Tab. 2 sind die verschiedenen Konstellationen zusammengestellt, die nicht im Text besprochenen Fälle hinzugefügt, der mögliche Fall 3 (Zielkosten = Plankosten oder $k_{Z, P}=k_{P, P}$ ) hingegen nicht aufgeführt, da der Zielkostenindex für diesen Fall zutreffende Informationen erzeugt. Dies lässt sich kurz erläutern: Im Fall a ist der Zielkostenindex größer als 1 und damit ist auch $a_{K}>b_{K}$, d. h., der Plankostenanteil der Komponente liegt richtigerweise über dem Zielkostenanteil. Im Fall b ist der Zielkostenindex gleich 1 und damit auch der Plankostenanteil gleich dem Zielkostenanteil. Im Fall c liegt der Zielkostenindex unter 1 und so müssen die Plankostenanteile kleiner als die Zielkostenanteile sein und daher ist auch $a_{K}<b_{K}$ korrekt. 
Tab. 3 Zahlenbeispiel zum modifizierten Zielkostendiagramm

\begin{tabular}{lllllll}
\hline & $\begin{array}{l}\text { Kostenanteil } \\
\left(\bar{a}_{K}\right)\end{array}$ & $\begin{array}{l}\text { Bedeutung } \\
\left(b_{K}\right)\end{array}$ & $\begin{array}{l}\text { Differenz } \\
\left(\bar{a}_{K}-b_{K}\right)\end{array}$ & $\begin{array}{l}\text { Plankosten } \\
\left(k_{P, K} ;\right. \\
G E / K E)\end{array}$ & $\begin{array}{l}\text { Zielkosten } \\
\left(k_{Z, K} ;\right. \\
G E / K E)\end{array}$ & $\begin{array}{l}\text { Zielkosten- } \\
\text { abweichung } \\
\left(\Delta k_{Z, K} ; G E / K E\right)\end{array}$ \\
\hline A & $\begin{array}{l}0,31 \\
(=138 / 450)\end{array}$ & 0,25 & $\begin{array}{l}0,06 \\
(=0,31-0,25)\end{array}$ & $\begin{array}{l}138,00 \\
(=0,23 \cdot 600)\end{array}$ & $\begin{array}{l}112,50 \\
(=0,25 \cdot 450)\end{array}$ & $\begin{array}{l}25,50 \\
(=138-112,50)\end{array}$ \\
$\mathrm{B}$ & $\begin{array}{l}0,63 \\
(=282 / 450)\end{array}$ & 0,50 & $\begin{array}{l}0,13 \\
(=0,63-0,50)\end{array}$ & $\begin{array}{l}282,00 \\
(=0,47 \cdot 600)\end{array}$ & $\begin{array}{l}225,00 \\
(=0,50 \cdot 450)\end{array}$ & $\begin{array}{l}57,00 \\
(=282-225)\end{array}$ \\
$\mathrm{C}$ & $\begin{array}{l}0,40 \\
(=180 / 450)\end{array}$ & 0,25 & $\begin{array}{l}0,15 \\
(=0,40-0,25)\end{array}$ & $\begin{array}{l}180,00 \\
(=0,30 \cdot 600)\end{array}$ & $\begin{array}{l}112,50 \\
(=0,25 \cdot 450)\end{array}$ & $\begin{array}{l}67,50 \\
(=180-112,50)\end{array}$ \\
Summe & 1,34 & 1,00 & 1,34 & $\begin{array}{l}600,00 \\
\left(k_{P, P} ; G E / P E\right)\end{array}$ & $\begin{array}{l}450,00 \\
\left(k_{Z, P} ; G E / P E\right)\end{array}$ & $\begin{array}{l}150,00 \\
\left(\Delta k_{Z, P} ; G E / P E\right)\end{array}$ \\
\hline
\end{tabular}

Die Fallanalyse zeigt, dass der Zielkostenindex für die Zielkostenkontrolle nicht geeignet ist. Überspitzt formuliert, erzeugt er nur dann mit Sicherheit verlässliche Informationen, wenn er nicht gebraucht wird, d. h., wenn die Plankosten gleich den Zielkosten sind. Daher finden sich in der Literatur zur Zielkostenkontrolle weiterentwickelte Konzepte, wie der Zielkostenindex alternativ zu berechnen ist.

Fischer u. Schmitz (1994) stellen ein erweitertes Zielkostenkontrolldiagramm vor, um die im vorigen Kapitel beschriebenen Probleme zu beseitigen. In ihrem Vorschlag berechnen sie einen modifizierten Plankostenanteil für die Komponente $\left(\bar{a}_{K}\right)$ nicht mehr auf Basis der Plankosten des Produkts, sondern auf Basis der Zielkosten des Produkts (vgl. Fischer u. Schmitz 1994, S. 428 f.; Coenenberg et al. 2007, S. 544 ff.).

$$
\bar{a}_{K}=\frac{k_{P, K}}{k_{Z, P}}
$$

In der Folge griffen eine Reihe von Autoren diesen Vorschlag auf, verzichteten aber aufgrund der Einsicht über die Unzulänglichkeit des Zielkostenindex konsequent auf die Berechnung in seiner ursprünglichen Form und erstellten ein modifiziertes Zielkostenkontrolldiagramm, bei dem auf der $y$-Achse der modifizierte Plankostenanteil $\left(\bar{a}_{K}\right)$ und auf der $x$-Achse weiterhin die Bedeutung $\left(b_{K}\right)$ abgetragen wird (vgl. Listl 1998, S. 196 ff.; Glaser 2002, S. 235). Diese Vorgehensweise soll kurz an dem bereits eingeführten Beispiel fortgesetzt werden (vgl. die Vorgehensweise z. B. in SchulteHenke 2008, S. 51 ff.).

Aufgrund der Berechnung der Kennzahlen des modifizierten Plankostenanteils und der Bedeutung ist das Zielkostenkontrolldiagramm konsequent auf die Zielkosten ausgerichtet. Die Ideallinie in Abb. 2 bleibt weiterhin die Winkelhalbierende und das Diagramm zeigt die Handlungsmöglichkeiten korrekt an: Alle Komponenten haben Kostenreduktionsbedarf und liegen über der Ideallinie.

In Tab. 3 fällt bei einem Vergleich der Werte auf, dass sich die Differenz zwischen dem modifizierten Plankostenanteil und der Bedeutung nicht in der komponentenbezogenen Abweichung widerspiegelt, da sie sich auf die Zielkosten des Produkts bezieht. Dies gilt dementsprechend für das modifizierte Zielkostenkontrolldiagramm: Trägt man eine vertikale Strecke vom jeweiligen Punkt zur Ideallinie ab, dann stellt ihre Länge die gleiche Differenz dar. Wenn der Wert von 0,06 für die Komponente A in Prozent ausgedrückt wird, dann bedeutet dies, dass die Zielkostenabweichung der 


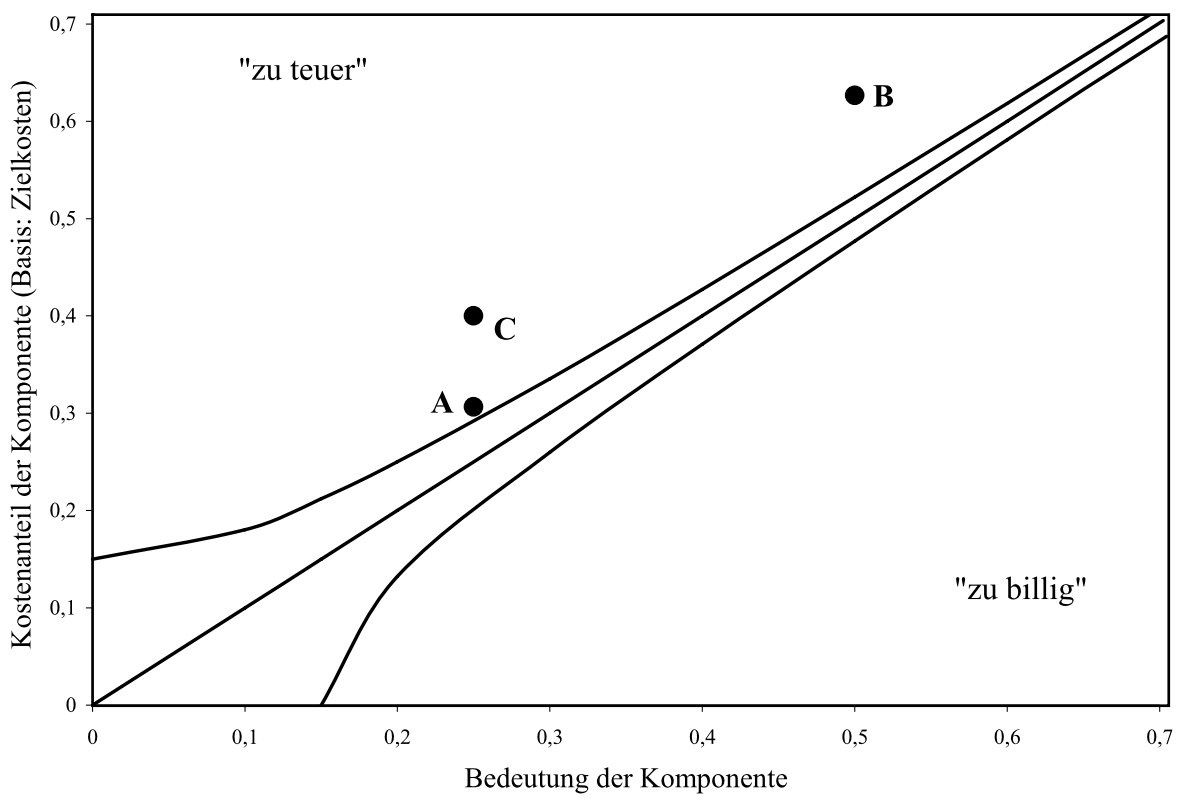

Abb. 2 Modifiziertes Zielkostenkontrolldiagramm

Komponente 6\% der Zielkosten des Produkts ausmacht (vgl. Meyer 2003, S. 240). Es lässt sich allerdings nicht erkennen, wie weit der relative Abstand zwischen den Plankosten der Komponente und den Zielkosten der Komponente ist. Es wird auch nicht recht klar, für welchen Rechnungszweck diese Information generiert wird, da als Kontrollobjekt zwar die Komponente angegeben wird, in der Kennzahl jedoch die Zielkosten des Produkts als Referenz verwendet werden. Konsequenter ist es, Informationen über Kostenabweichungen für das Produkt und die Komponenten zur Verfügung zu stellen.

\section{Die relative Zielkostenabweichung}

Wenn eine relative Information zur Zielkostenabweichung für das Produkt und die einzelnen Komponenten konstruiert werden soll, bietet es sich an, direkt an die Zielkostenabweichung anzuknüpfen, indem die Relation zwischen Plankosten und Zielkosten gebildet wird (vgl. Betz 1995, S. 613; Monden 1999, S. 256 f.; Clifton et al. 2004, S. 65; Hoque et al. 2005, S. 1621; Kreuz 2005, S. 148; Schild 2005, S. 308; Kremin-Buch 2007, S. 141; Winkler 2008, S. 110; Brühl 2009, S. 210). ${ }^{5}$

\footnotetext{
${ }^{5}$ In den angegebenen Quellen wird die Kennzahl zum Teil in umgekehrter Relation gebildet und unterschiedlich bezeichnet: Hoque et al. (2005) sowie Monden (1999) verwenden „Wert" und Clifton et al. (2004) ,Wertindex“, Betz (1995), Schild (2005), Kreuz (2005), Kremin-Buch (2007) und Winkler (2008) benennen sie weiterhin als „Zielkostenindex“ oder „,modifizierter Zielkostenindex“, wobei Kreuz (2005) und Kremin-Buch (2007) zwischen absoluten und relativen Zielkostenindices unterscheiden.
} 
Tab. 4 Zahlenbeispiel zur relativen Zielkostenabweichung

\begin{tabular}{|c|c|c|c|c|c|c|}
\hline & $\begin{array}{l}\text { Kostenanteil } \\
\left(a_{K}\right)\end{array}$ & $\begin{array}{l}\text { Bedeutung } \\
\left(b_{K}\right)\end{array}$ & $\begin{array}{l}\text { Relative Zielko- } \\
\text { stenabweichung } \\
\left(r Z A_{K}\right)\end{array}$ & $\begin{array}{l}\text { Plankosten } \\
\left(k_{P, K} ;\right. \\
G E / K E)\end{array}$ & $\begin{array}{l}\text { Zielkosten } \\
\left(k_{Z, K} ;\right. \\
G E / K E)\end{array}$ & $\begin{array}{l}\text { Zielkosten- } \\
\text { abweichung } \\
\left(\Delta k_{Z, K} ; G E / K E\right)\end{array}$ \\
\hline A & 0,23 & 0,25 & $\begin{array}{l}1,23 \\
(=138 / 112,50)\end{array}$ & $\begin{array}{l}138,00 \\
(=0,23 \cdot 600)\end{array}$ & $\begin{array}{l}112,50 \\
(=0,25 \cdot 450)\end{array}$ & $\begin{array}{l}25,50 \\
(=138-112,50)\end{array}$ \\
\hline B & 0,47 & 0,50 & $\begin{array}{l}1,25 \\
(=282 / 225)\end{array}$ & $\begin{array}{l}282,00 \\
(=0,47 \cdot 600)\end{array}$ & $\begin{array}{l}225,00 \\
(=0,50 \cdot 450)\end{array}$ & $\begin{array}{l}57,00 \\
(=282-225)\end{array}$ \\
\hline $\mathrm{C}$ & 0,30 & 0,25 & $\begin{array}{l}1,60 \\
(=180 / 112,50)\end{array}$ & $\begin{array}{l}180,00 \\
(=0,30 \cdot 600)\end{array}$ & $\begin{array}{l}112,50 \\
(=0,25 \cdot 450)\end{array}$ & $\begin{array}{l}67,50 \\
(=180-112,50)\end{array}$ \\
\hline Summe & 1,00 & 1,00 & & $\begin{array}{l}600,00 \\
\left(k_{P, P} ; G E / P E\right)\end{array}$ & $\begin{array}{l}450,00 \\
\left(k_{Z, P} ; G E / P E\right)\end{array}$ & $\begin{array}{l}150,00 \\
\left(\Delta k_{Z, P} ; G E / P E\right)\end{array}$ \\
\hline
\end{tabular}

$$
r Z A_{P}=\frac{k_{P, P}}{k_{Z, P}} \quad \text { und } \quad r Z A_{K}=\frac{k_{P, K}}{k_{Z, K}}
$$

Die relative Zielkostenabweichung des Produkts $\left(r Z A_{P}\right)$ und die relative Zielkostenabweichung der Komponente $\left(r Z A_{K}\right)$ beruhen im Gegensatz zum Zielkostenindex auf den Kostenbeträgen. Sie sind so konstruiert, dass sie für die beiden Rechnungszwecke Überprüfung der Zielkostenerreichung und Überprüfung der Kosten-NutzenRelationen gleichzeitig Informationen liefern. Im Falle, dass die Plankosten gleich den Zielkosten der Komponente sind, ist ihre relative Zielkostenabweichung gleich 1 und zeigt damit die ideale Kosten-Nutzen-Relation an. Zur Illustration der relativen Zielkostenabweichung wird in Tab. 4 das Zahlenbeispiel aus Tab. 1 fortgesetzt.

In Tab. 4 wird ausgewiesen, dass alle Komponenten einen Kostenreduktionsbedarf haben, da für jede Komponente die relative Zielkostenabweichung größer als 1 ist. Es wird eine relative Abweichung angezeigt, die sich auch in Prozent ausdrücken lässt (vgl. Schild 2005, S. 308). Ein Wert von 1,23 (Komponente A) drückt aus, dass die Plankosten $23 \%$ über den Zielkosten liegen; die relative Zielkostenabweichung des Produkts beträgt 1,33 (=600/450). Wenn die Berechnung in Prozent erfolgen soll, muss in Formel (12) der Quotient mit 100 multipliziert werden; die Kennzahl erhält dann jeweils die Dimension [\%].

Das zur relativen Zielkostenabweichung passende Diagramm wird konstruiert (Abb. 3), indem auf der $x$-Achse die Zielkosten und auf der $y$-Achse die Plankosten abgetragen werden (vgl. Seidenschwarz et al. 1997, S. 118; Mussnig 2001, S. 271). Dementsprechend sind die Untergrenzen $\left(y_{U G}, G E / K E\right)$ und Obergrenzen $\left.y_{O G}, G E / K E\right)$ zu bilden (vgl. Glaser 2006, S. 218).

$$
y_{U G}=k_{Z, P} \cdot \sqrt{\max \left\{b_{K}^{2}-\alpha^{2}, 0\right\}} \text { und } y_{O G}=k_{Z, P} \cdot \sqrt{b_{K}^{2}+\alpha^{2}}
$$

Dies soll beispielhaft für die Komponente A erfolgen:

$$
450 \cdot \sqrt{0,25^{2}-0,15^{2}}<112,50<450 \cdot \sqrt{0,25^{2}+0,15^{2}}
$$

Die Werte für die Komponenten A $\left(y_{O G}=131,20, K_{P, K}=138\right)$, B $\left(y_{O G}=235\right.$, $\left.K_{P, K}=282\right)$ und C $\left(y_{O G}=131, K_{P, K}=180\right)$ liegen außerhalb der Zielkostenzone und zeigen Kostenreduktionsbedarf an. Die Winkelhalbierende stellt auch in diesem Diagramm die Ideallinie dar. Mit einer vertikalen Verbindung zwischen ihr und den 


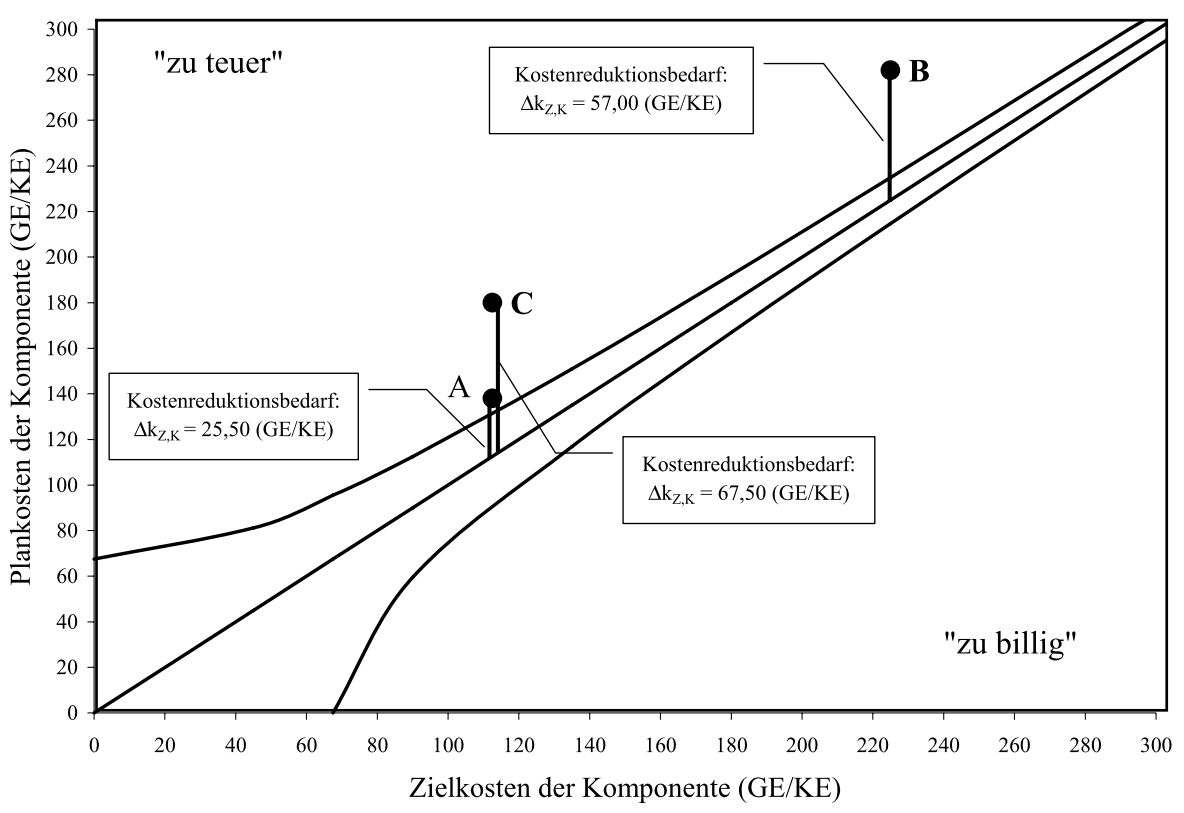

Abb. 3 Zielkostenkontrolldiagramm auf Basis von Kostenbeträgen

Punkten der Komponenten wird der Betrag der notwendigen Kostenreduktion der Komponente abgetragen (s. Abb. 3).

\section{Diskussion}

Abschließend sollen die vorgestellten Varianten von Kennzahlen und ihre entsprechenden Diagramme kurz vergleichend diskutiert werden. So enthält das Diagramm auf Basis der relativen Zielkostenabweichung bezüglich der Kosten-Nutzen-Relation die gleiche Information wie das modifizierte Zielkostendiagramm, denn die Reihenfolge der Komponenten und auch die relative Lage innerhalb der Zielkostenkontrolldiagramme sind gleich (s. Abb. 2 und 3). Im modifizierten Zielkostenkontrolldiagramm sind die $x$-Werte die Bedeutungen und die $y$-Werte die Plankosten der Komponente bezogen auf die Zielkosten des Produkts; ein Index auf dieser Basis lautet wie folgt (vgl. Schild 2005, S. 308):

$$
\frac{\bar{a}_{K}}{b_{K}}
$$

Dies lässt sich zur relativen Zielkostenabweichung umformen:

$$
\frac{\bar{a}_{K}}{b_{K}}=\frac{k_{P, K}}{k_{Z, P}} \cdot \frac{k_{Z, P}}{k_{Z, K}}=\frac{k_{P, K}}{k_{Z, K}}
$$

Beide Vorgehensweisen zeigen daher die Handlungsmöglichkeiten korrekt an. Ein weiterer Vorteil gegenüber dem Zielkostenindex: Müssen aufgrund neuer Schätzun- 
Tab. 5 Vorschlag zu den Kennzahlen für die Zielkostenkontrolle

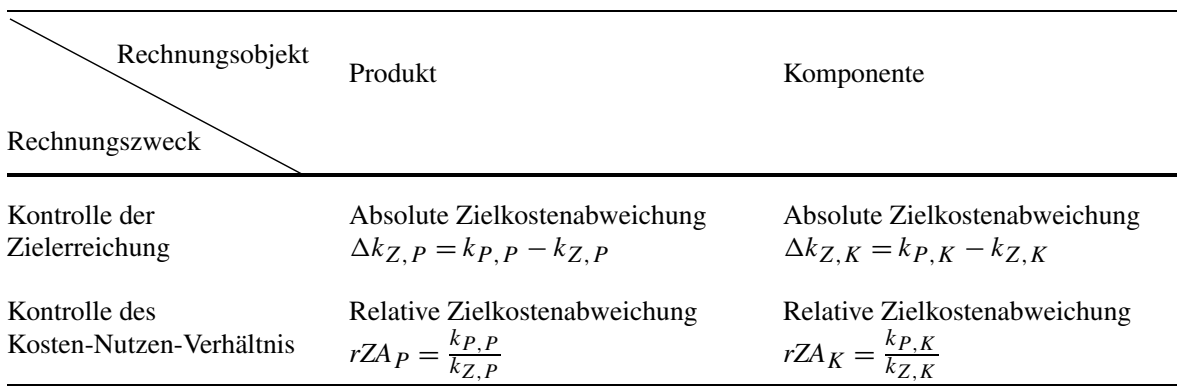

gen die Plankosten einzelner Komponenten modifiziert werden, bleiben die Kennzahlen der anderen Komponenten gleich. Der Zielkostenindex variiert hingegen für alle Komponenten, wenn in einer Komponente Kostenänderungen auftreten, d. h., auch wenn an einer Komponente überhaupt keine Veränderungen vorgenommen werden, ändert sich ihr Zielkostenindex (vgl. Listl 1998, S. 194).

Die relative Zielkostenabweichung hat allerdings den Vorteil, dass sie zweckgerichtet Informationen über den Kostenreduktions- oder den Qualitätsverbesserungsbedarf der Komponente liefert, die konsistent zur Zielkostenabweichung in Geldeinheiten sind. Des Weiteren hat das Zielkostenkontrolldiagramm auf Basis der relativen Kostenabweichung und Kostenbeträge den gleichen Vorteil wie die Kennzahl relative Zielkostenabweichung. Es visualisiert die Sachverhalte für beide relevanten Rechnungszwecke: Es zeigt einerseits die relative Lage bezüglich der Ideallinie und damit die Kosten-Nutzen-Relation und andererseits mittels einer vertikalen Linie vom Punkt der Komponente im Diagramm zur Ideallinie die absoluten Abweichungsbeträge. Deswegen ist es nur dann sinnvoll, zwei Zielkostenkontrolldiagramme zu konstruieren (wie z. B. in Seidenschwarz et al. 1997, S. 118 vorgeschlagen), wenn die Kostenbeträge der Komponenten stark streuen und das Zielkostenkontrolldiagramm unübersichtlich wird (vgl. Listl 1998, S. 198).

Werden die vier Kennzahlen (Tab. 5) mit dem Zielkostenkontrolldiagramm auf Basis der Kostenbeträge verbunden, stellen sie ein in sich konsistentes Kennzahlengefüge dar, aus dem sich die drei Handlungsmöglichkeiten - Kostenreduktion, Qualitätsverbesserung und kein Handlungsbedarf - für das Produkt und die Komponenten widerspruchsfrei ableiten und visualisieren lassen.

Danksagung Für kritische Anmerkungen zu einer ersten Fassung des Artikels danke ich meinem Kollegen Prof. Dr. Robert Wilken, meinem wissenschaftlichen Mitarbeiter Dipl.-Kfm. Mathias Orth, Herrn Dipl.-Kfm. Marco Reimer und zwei anonymen Gutachtern sowie dem Herausgeber Prof. Dr. Uwe Götze.

\section{Literatur}

Ansari, S., Bell, J., \& Okano, H. (2007). Target costing: uncharted research territory. In C.S. Chapman, A.G. Hopwood, M.D. Shields, \& S. Amsterdam (Hrsg.), Handbook of Management Accounting Research (Bd. 2, S. 507-530).

Betz, S. (1995). Die Erfahrungskurve als Instrument der Zielkostenspaltung. Betriebswirtschaftliche Forschung und Praxis, 47, 609-625. 
Brühl, R. (2009). Controlling. Grundlagen des Erfolgscontrollings (2. Aufl.). München.

Brühl, R. (2010). Kennzahlen für die Zielkostenkontrolle: vom Zielkostenindex zur relativen Zielkostenabweichung (Workingpaper). ESCP Europe Wirtschaftshochschule Berlin, Berlin (im Erscheinen).

Clifton, M. B., Bird, H. M. B., \& Albano, R. E. (2004). Target costing. Market-driven product design. New York.

Coenenberg, A. G., Fischer, T. M., \& Günther, T. (2007). Kostenrechnung und Kostenanalyse (6. Aufl.). Stuttgart.

Cooper, R., \& Slagmulder, R. (2005). Target costing for new product development. In R.L. Weil, M. Maher (Hrsg.), Handbook of cost management (2. Aufl., S. 243-270).

Fischer, T. M., \& Schmitz, J. A. (1994). Informationsgehalt und Interpretation des Zielkostenkontrolldiagramms im Target Costing. In Kostenrechnungspraxis (S. 427-433).

Franz, K.-P. \& Kajüter, P. (2002). Kostenmanagement in Deutschland. In K.-P. Franz \& P. Kajüter (Hrsg.), Kostenmanagement: Wertsteigerung durch systematische Kostensteuerung (2. Aufl., S. 569-585). Stuttgart.

Freidank, C.-C., \& Zaeh, P. (1997). Spezialfragen des Target Costing und des Kostenmanagements. In C.-C. Freidank, U. Götze, B. Huch, \& J. Weber (Hrsg.), Kostenmanagement. Aktuelle Konzepte und Anwendungen (S. 233-274). Berlin.

Glaser, H. (2002). Target costing as a strategic controlling instrument. In C. Scholz \& J. Zentes (Hrsg.), Strategic management. A European approach (S. 221-239). Wiesbaden

Glaser, H. (2006). Target Costing als Controllinginstrument des Mittelstand. Controlling, 18, 215-219.

Götze, U. (2007). Kostenrechnung und Kostenmanagement (4. Aufl.). Berlin.

Götze, U. \& Linke, C. (2008). Interne Unternehmensrechnung als Instrument des marktorientierten Zielkostenmanagements - ausgewählte Probleme und Lösungsansätze. ZfP, 19, 107-132.

Hoque, M., Akter, M., \& Monden, Y. (2005). Concurrent engineering - A compromising approach to develop a feasible and customer-pleasing product. International Journal of Production Research, 43, 1607-1624.

Kajüter, P. (2005). Kostenmanagement in der deutschen Unternehmenspraxis. Zeitschrift für betriebswirtschaftliche Forschung, 57, 79-100.

Kremin-Buch, B. (2007). Strategisches Kostenmanagement. Grundlagen und moderne Instrumente (4. Aufl.). Wiesbaden.

Kreuz, C. (2005). Kundenorientierte Unternehmensrechnung. Planung, Steuerung und Kontrolle von Kundenwerten. Wiesbaden.

Listl, A. (1998). Target Costing zur Ermittlung der Preisuntergrenze: entscheidungsorientiertes Kostenmanagement dargestellt am Beispiel der Automobilzulieferindustrie. Frankfurt am Main.

Meyer, J. W. (2003). Produktinnovationserfolg und Target Costing. Wiesbaden.

Monden, Y. (1999). Wege zur Kostensenkung. München.

Mussnig, W. (2001). Dynamisches Target Costing. Von der statischen Betrachtung zum strategischen Management der Kosten. Wiesbaden.

Noltemeier, S. (2003). Zur Konzeption monetärer Anreizsysteme für das Target Costing. Wiesbaden.

Schild, U. (2005). Lebenszyklusrechnung und lebenszyklusbezogenes Zielkostenmanagement. Wiesbaden.

Schulte-Henke (2008). Kundenorientiertes Target Costing und Zuliefererintegration für komplexe Produkte. Entwicklung eines Konzepts für die Automobilindustrie. Wiesbaden.

Schweitzer, M., \& Küpper, H.-U. (2008). Systeme der Kosten- und Erlösrechnung (9. Aufl.). München.

Seidenschwarz, W., Esser, J., Niemand, S., \& Rauch, M. (1997). Target Costing: Auf dem Weg zum marktorientierten Unternehmen. In K.-P. Franz \& P. Kajüter (Hrsg.), Kostenmanagement. Wertsteigerung durch systematische Kostensteuerung (S. 101-126). Stuttgart.

Tanaka, M. (1985). New approach to the function evaluation system in value engineering. International Journal of Production Research, 23, 625-637.

Tanaka, M. (1989). Cost planning and control system in the design phase of a new product. In Y. Monden \& M. Sakurai (Hrsg.), Japanese management accounting. A world class approach to profit management (S. 49-71). Cambridge.

Winkler, A. (2008). Target Costing auf Basis unvollkommener Informationen. Zu den Möglichkeiten und Grenzen fuzzy-basierter Ansätze im Target Costing. Hamburg. 